\title{
Modificação técnica na cirurgia da estenose aórtica supravalvar
}

\author{
Luiz Carlos Bento de SOUZA*, Paulo CHACCUR*, Jarbas J. DINKHUYSEN*, M. A. FONTES \\ F. FONTES*, Camilo ABDULMASSIH NETO*, Antoninho S. ARNONI**, Maria Virgínia D. SILVA**, leda

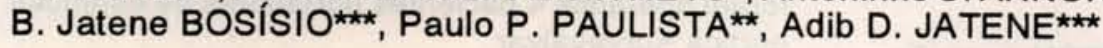

SOUZA, L. C. B.; CHACCUR, P.; DINKHUYSEN, J. J.; FONTES, M. A.; FONTES, V. F.; ABDULMASSIH NETO, C.; ARNONI, A. S.; SILVA, M. V. D.; BOSISIO, I. B. J.; PAULISTA, P. P.; JATENE, A. D. Modificaçāo técnica na cirurgia da estenose aórtica supravalvar. Rev. Bras. Cir. Cardiovasc., 7(2):121-126, 1992.

RESUMO: O tratamento cirúrgico convencional da estenose aórtica supravalvar caracteriza-se pela ampliaçāo de um ou mais seios de Valsalva, utilizando-se retalhos de material protético, com ou sem secção transversal da aorta. Uma possível limitaçăo dos resultados deste procedimento é o fato do endurecimento ou calcificação do enxerto, dificultando o desenvolvimento da raíz da aorta, mormente quando a cirurgia é realizada em criaças. Para evitar essa complicação, desenvolvemos uma modificação técnica que, efetivamente, amplia o diâmetro da raíz da aorta, sem o uso de material protético, aproveitando apenas o tecido sadio da parede da aorta ascendente, para reconstruir e ampliar os seios de Vasalva. Com esta técnica foram operados 4 pacientes com diagnósticos clínico e hemodinâmico de estenose aórtica supravalvar, com os seguintes dados clínicos: idades, 1 ano e 11 meses, 3 anos e 9 meses, 15 e 38 anos, sexo masculino três casos, peso corporal de 10, 12, 27 e $56 \mathrm{~kg}$. Dois pacientes tinham dispnéia, um palpitaçōes freqüentes e outro era assintomático. Os gradientes entre a cavidade livre do ventrículo esquerdo e aorta ascendente eram de 50 , 70,100 e $100 \mathrm{mmHg}$. Os pacientes foram operados com o auxílio da circulação extracorpórea, de hipotermia moderada e emprego de cardioplegia cristalóide. A aorta ascendente foi dissecada em toda sua extensão, preferindo-se canular a artéria femoral para o retorno arterial, a fim de liberar a aorta ascendente para o procedimento. Após transecçāo total da aorta e ressecçāo do tecido fibrótico estenosante, foram feitas incisōes na borda livre até o fundo dos seios de Valsalva. Foram realizadas incisōes longitudinais na porçāo distal da aorta, nas regiöes correspondentes aos postes comissurais da valva aórtica, de tal forma que, durante a reconstrução por sutura direta, um segmento da parede aórtica ficasse ampliando um seio de Valsalva, obtendo-se, assim, uma raíz de aorta de aspecto anatômico e dimensōes normais. Todos os casos, tiveram evoluçâo favorável. Atualmente, com um período de pós-operatório de até seis meses, estão assintomáticos

DESCRITORES: estenose aórtica, supravalvar, cirurgia; valva aórtica, cirurgia.

\section{INTRODUÇĀO}

A estenose aórtica supravalvar é interpretada como uma obstruçāo congênita, localizada ou difusa, iniciando-se imediatamente acima da valva aórtica e dos óstios coronarianos.

Entre as deformidades que produzem obstáculo à livre ejeção do ventrículo esquerdo (VE) e situadas próximas à valva aórtica, ela representa, segundo PETERSON et alii ${ }^{10} 5,8 \%$, BEUREN et alii ${ }^{~}$ $3,2 \%$ e HANCOCK ${ }^{6} 0,6 \%$ dos casos de estenose aórtica.

A correçāo cirúrgica dessa lesāo foi realizada pela primeira vez em agosto de 1956, por McGOON et aliii ${ }^{8}$, usando a técnica de alargamento da raíz da

Trabalho realizado do Instituto Dante Pazzanese de Cardiologia e no Hospital do Coraçáo da Associação do Santório Sírio. Sảo Paulo, SP, Brasil. Apresentado ao $19^{\circ}$ Congresso Nacional de Cirurgia Cardíaca. Sáo Paulo, SP, 7 a 9 de março de 1992.

- Do Instituto Dante Pazzanese de Cardiologia e do Hospital do Coração da Associação do Sanatório Sírio.

* Do Instituto Dante Pazzanese de Cardiologia.

* Do Hospital do Coraçảo da Associação do Sanatório Sírio

Endereço para separatas: Luiz Carlos Bento de Souza. Rua Desembargador Elizeu Guilherme, 123. 04004 Paraíso. Sảo Paulo, SP, Brasil. 
SOUZA, L. C. B.; CHACCUR, P.; DINKHUYSEN, J. J.; FONTES, M. A.; FONTES, V. F.; ABDULMASSIH NETO, C.; ARNONI, A. S.; SILVA, M. V. D.; BOSíSIO, I. B. J.; PAULISTA, P. P.; JATENE, A. D. - Modificação técnica na cirurgia da estenose aórtica supravalvar. Rev. Bras. Cir. Cardiovasc., 7(2):121-126, 1992.

aorta na regiāo correspondente ao seio não coronariano, com material protético.

Posteriormente, em 1977, DOTY et alii ${ }^{4}$ chamaram a atenção para a necessidade de se fazer a aortoplastia estendida, ampliando, também, o seio coronariano direito.

A primeira referência sobre o uso de secção transversal da aorta com ressecção do tecido fibroso estenosante e reaproximação dos cotos aórticos foi de HARA et alii ${ }^{7}$, em 1962.

Mais recentemente, em 1990 , BROM ${ }^{3}$ propôs a secçăo transversal da aorta, seguida de uma incisão longitudinal desde a borda livre do orifício estenótico até o seio de Valsalva, nos três seios, ampliando-os com retalhos de pericárdio bovino. Entre nós, MURAD et alii ${ }^{\circ}$ apresentaram seus resultados com essa técnica.

Atualmente, temos uma dupla opção técnica para a cirurgia da estenose aórtica supravalvar, na forma localizada: a primeira representada pela ampliação longitudinal da aorta, em um ou dois seios coronarianos, e a outra após secção transversa, ampliação dos seios coronarianos com retaIhos de pericárdio individualmente seguida de anastomose das duas porçōes da aorta.

A finalidade do presente trabalho é apresentar uma modificaçāo técnica onde, após a secção transversal da aorta, os seios de Valsalva são ampliados pela própria parede da aorta ascendente preparada para essa finalidade. Desta forma não teremos material protético fazendo parte da correção e, por conseguinte, sem eventuais complicaçōes pelo comportamento desses tecidos, principalmente em crianças.

Sob o ponto de vista cirúrgico, existem dois tipos de estenose aórtica supravalvar: a localizada, interessando somente a área acima das válvulas e

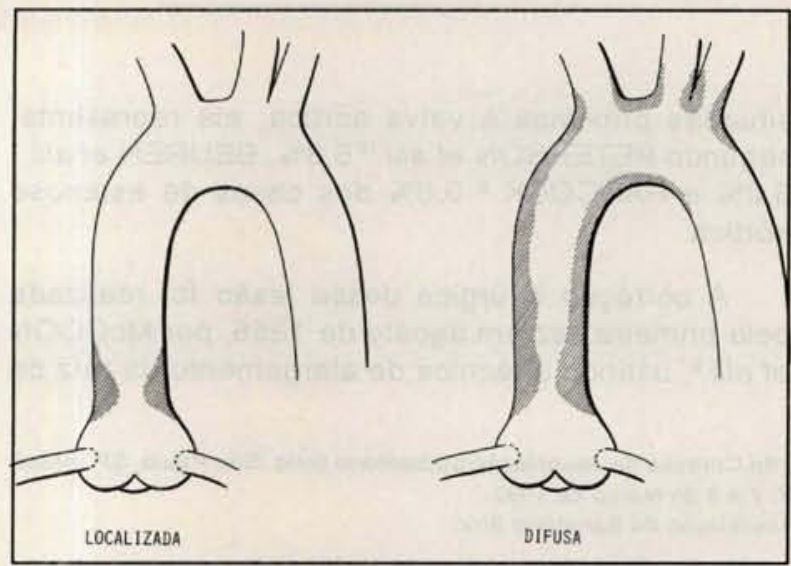

Fig.1 - Representaçáo esquemática das duas formas da estenose aórtica supravalvar: localizada e difusa. os óstios coronarianos, e a difusa, englobando aorta ascendente, croça e, eventualmente, os troncos supra-aórticos. Referiremo-nos, no presente trabaIho, ao tratamento cirúrgico da forma localizada (Figura 1).

\section{CASUÍSTICA E MÉTODOS}

No período entre de 1991 e março de 1992, foram operados 4 pacientes portadores de estenose aórtica supravalvar.

Três $(75 \%)$ eram do sexo masculino, com idades de 1 ano e 1 meses, 3 anos e 9 meses, 15 anos e 38 anos. O peso corporal foi de $10,12,27$ e 56 kg. O paciente de 38 anos e, portanto, com maior peso corporal, era do sexo feminino.

Dois pacientes apresentavam dispnéia (GFI-1, GFIII-1), um tinha palpitaçōes freqüentes e outro era assintomático.

Os 3 pacientes mais jovens apresentavam sinais da síndrome descrita, primeiramente, por WILLIAMS et alii ${ }^{14} e$, posteriormente, por BEUREN et alii $^{2}$, onde aparência facial (elfin face), graus variáveis de retardo mental, problemas odontológicos e estenoses periféricas em ramos da artéria pulmonar representam as anormalidades mais sugestivas.

A paciente de 38 anos de idade não mostrava sinais dessa sindrome, parecendo ser um caso da forma esporádica da enfermidade.

Todos os casos apresentavam sopro sistólico em área aórtica, algum aumento da área cardíaca e sobrecarga ventricular esquerda ao eletrocardiograma.

Os achados anatômicos obtidos pelo ecocardiograma foram concordantes com os apresentados pelo estudo hemodinâmico (Figura 2).

Os gradientes entre a cavidade livre do ventriculo esquerdo e a aorta ascendente foram de 50 , 70,100 e $100 \mathrm{mmHg}$, respectivamente.

Não havia defeitos cardíacos associados; somente no caso de número 3 ( 15 anos, masculino) foi encontrada associação de pequeno refluxo aórtico.

A primeira operação com esta técnica foi realizada em 01/10/91.

Através de esternotomia mediana longitudinal, são expostos o coração e os vasos da base. Em todos os casos foi evidente a observação da forma em ampulheta da raíz da aorta, que foi dissecada em toda sua extensão, até a emergência do tronco arterial braquiocefálico. 
SOUZA, L. C. B.; CHACCUR, P.; DINKHUYSEN, J. J.; FONTES, M. A.; FONTES, V. F.; ABDULMASSIH NETO, C.; ARNONI-A. S.; SILVA, M. V. D.; BOSÍSIO, I. B. J.; PAULISTA, P. P.; JATENE, A. D. - Modificação técnica na cirurgia da estenose aórtica supravalvar. Rev. Bras. Cir. Cardiovasc., $7(2): 121-126,1992$.

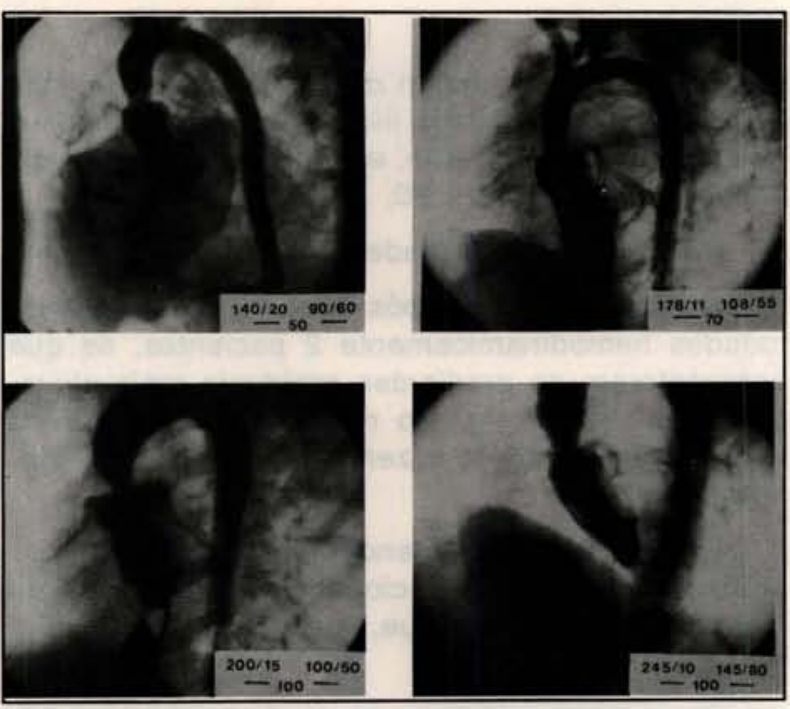

Fig. 2 - Ventriculografia esquerda em OAE dos 4 casos, acompanhada das pressóes de VE, Ao e gradiente sistólico.

É dissecada e canulada a artéria femoral comum para o retorno arterial, a fim de podermos ter livre a aorta ascendente para o procedimento.

A circulaçāo extracorpórea foi empregada com hipotermia sistêmica de $28^{\circ} \mathrm{C}$ e indução de cardioplegia com solução cristalóide fria e hipercalêmica infundida na raíz da aorta.

A aorta é transeccionada imediatamente acima da área estenótica.

A porção proximal contendo o orifício estenótico impede a visibilização adequada do interior dos seios da Valsalva, pelo que iniciamos uma incisão vertical da borda do orifício em direção ao fundo do seio não coronariano. Isto permite uma boa apresentação para identificação dos óstios coronarianos. Em seguida, realiza-se uma segunda incisão vertical no seio coronariano direito, que, pela visão do cirurgião, deverá passar à esquerda do óstio coronariano direito.

A terceira incisāo, praticada no seio coronariano esquerdo, deve ser conduzida à direita do óstio coronariano, evitando, assim, lesão no tronco da coronária esquerda, que passa posteriormente ao seio de Valsalva (Figura 3 ).

$\mathrm{Na}$ porçāo distal da aorta previamente dissecada, são feitas incisōes longitudinais, que têm início no local onde os postes comissurais, na porção proximal, se protejam no orifício da aorta distal. A profundidade dessas incisōes é a suficiente para transformar o orifício da aorta distal, em uma formação com três prolongamentos cuja parte mais proeminente deverá alcançar o fundo da incisão previamente feita no seio de Valsalva correspondente (Figura 3).

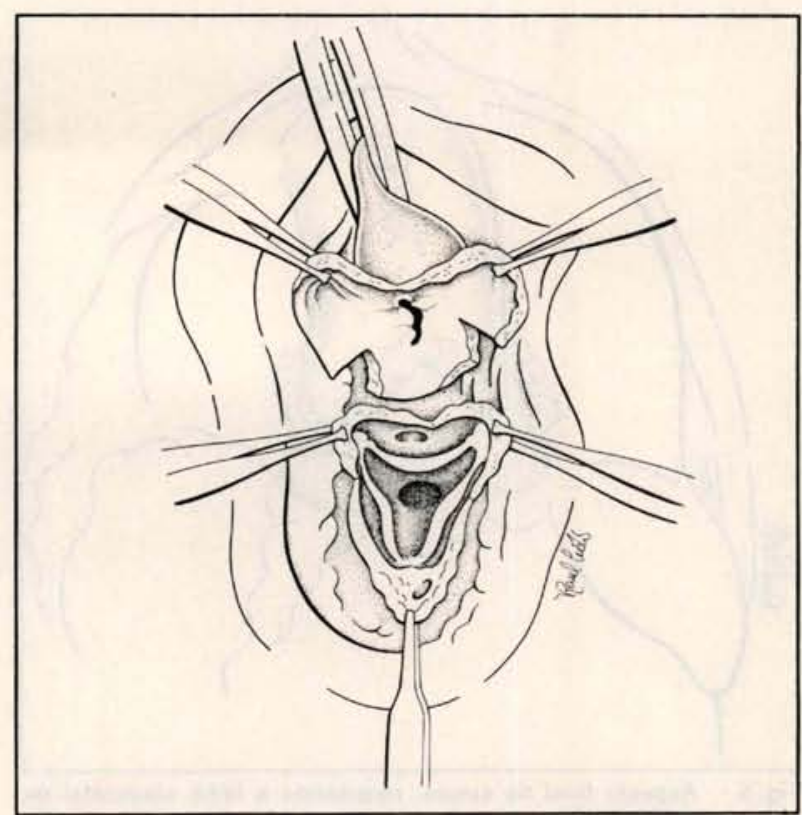

Fig. 3 - Incisóes feitas nos seios de Valsalva proximalmente e na aorta distalmente.

Com fio de monofilamento $5-0$, processa-se a sutura da aorta em toda a circunferência e que tomará um aspecto sinuoso, subindo ao nível dos postes comissurais e descendo ao fundo dos seios de Valsalva (Figuras 4 e 5 ). A eliminação do ar é feita por uma contra-abertura longitudinal na aorta ascendente.

O tempo de perfusāo cardiopulmonar variou de

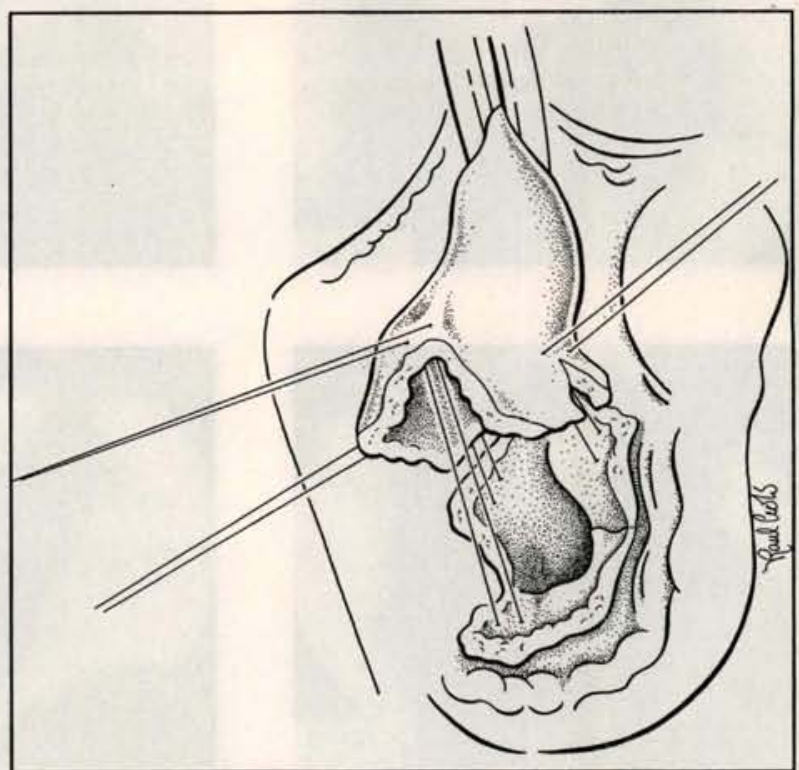

Fig. 4 - Colocam-se pontos de reparo nos postes comissurais, que seráo passados nos ângulos das incisōes da aorta distal. 
SOUZA, L. C. B.; CHACCUR, P.; DINKHUYSEN, J. J.; FONTES, M. A.; FONTES, V. F.; ABDULMASSIH NETO, C.; ARNONI, A. S.; SILVA, M. V. D.; BOSíSIO, I. B. J.; PAULISTA, P. P.; JATENE, A. D. - Modificaçāo técnica na cirurgia da estenose aórtica supravalvar. Rev. Bras. Cir. Cardiovasc., 7(2):121-126, 1992.

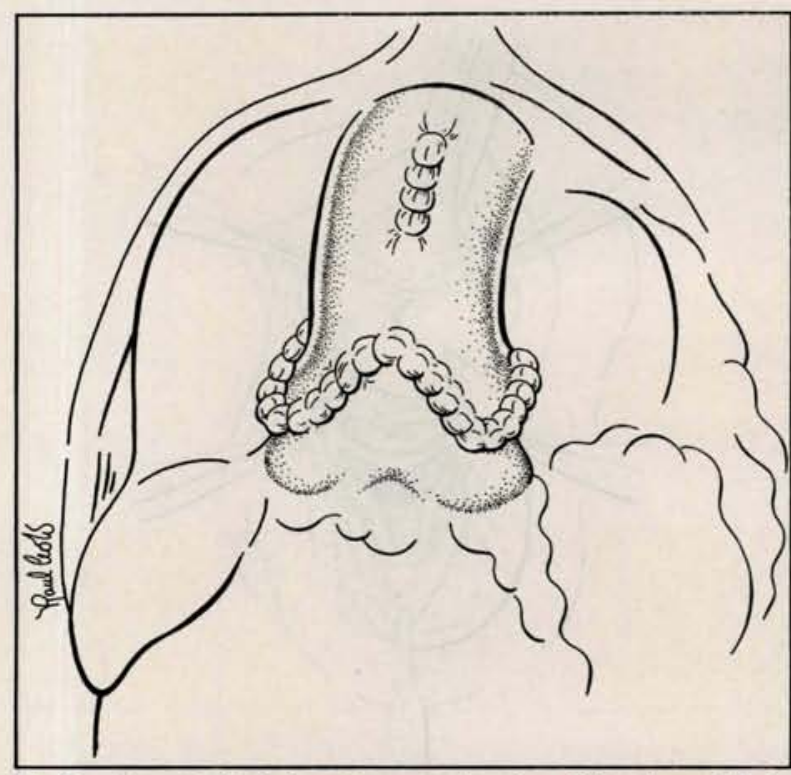

Fig. 5 - Aspecto final da sutura, mostrando a linha sinusoidal da anastomose.

50 a 75 minutos, com média de 62,5 minutos. 0 tempo de pinçamento aórtico variou de 40 a 50 minutos, com média de 44,2 minutos.

Todos os pacientes saíram da circulação extracorpórea em boas condiçōes hemodinâmicas.

\section{RESULTADOS}

Antes do fechamento do tórax, foram medidas as pressōes da cavidade livre do ventrículo esquerdo e da aorta ascendente, e os gradientes residuais encontrados foram de $20,20,35$ e $40 \mathrm{mmHg}$.

Não houve mortalidade hospitalar.

No primeiro mês de pós-operatório, foram reestudados hemodinamicamente 2 pacientes, os que apresentaram os gradientes residuais mais elevados, 35 e $40 \mathrm{mmHg}$. No reestudo, os gradientes caíram para $10 \mathrm{mmHg}$ e zero, respectivamente (Figuras 6 e 7).

Esse fato deixa a entender que esses pequenos gradientes estariam relacionados à hipertrofia da via de saída do $V E$, que, com a evolução, têm tendência a regredir.

Os pacientes evoluem em período pós-operatório de um a seis meses, estando todos assintomáticos.

\section{COMENTÁRIOS}

O tratamento cirúrgico, da forma localizada, da estenose aórtica supravalvar compreende, fundamentalmente, dois tipos de procedimentos: as ampliaçōes longitudinais simples ou duplas da área

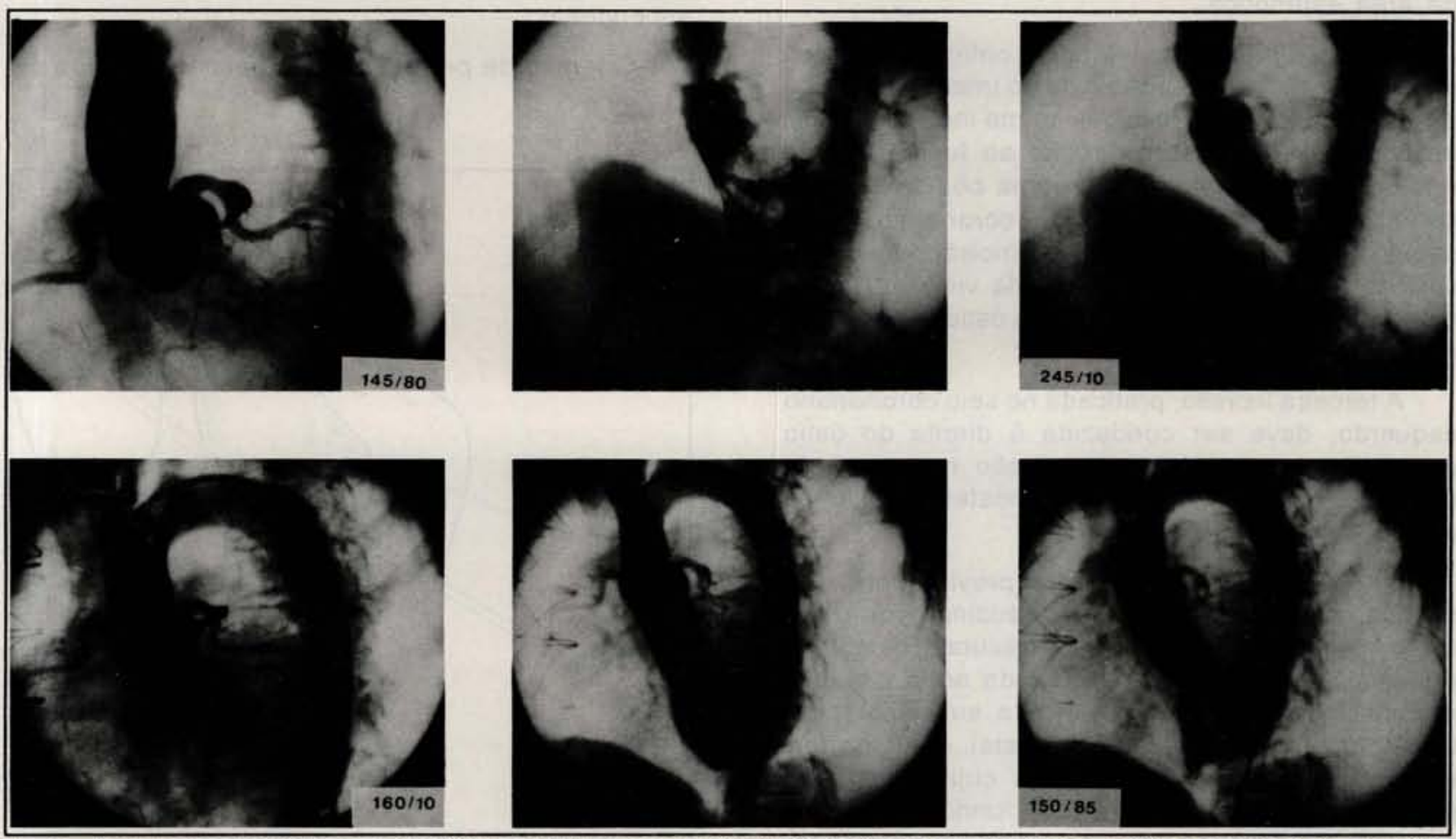

Fig. 6 - Aortograma e ventriculograma em diástole e sístole do caso de n³. A série horizontal superior é pré-operatória e a inferior é pós -operatória (20 dias) 
SOUZA, L. C. B.; CHACCUR, P.; DINKHUYSEN, J. J.; FONTES, M. A.; FONTES, V. F.; ABDULMASSIH NETO, C.; ARNONI, A. S.; SILVA, M. V. D.; BOSÍSIO, I. B. J.; PAULISTA, P. P.; JATENE, A. D. - Modificaçāo técnica na cirurgia da estenose aórtica supravalvar. Rev. Bras. Cir. Cardiovasc., 7(2):121-126, 1992.

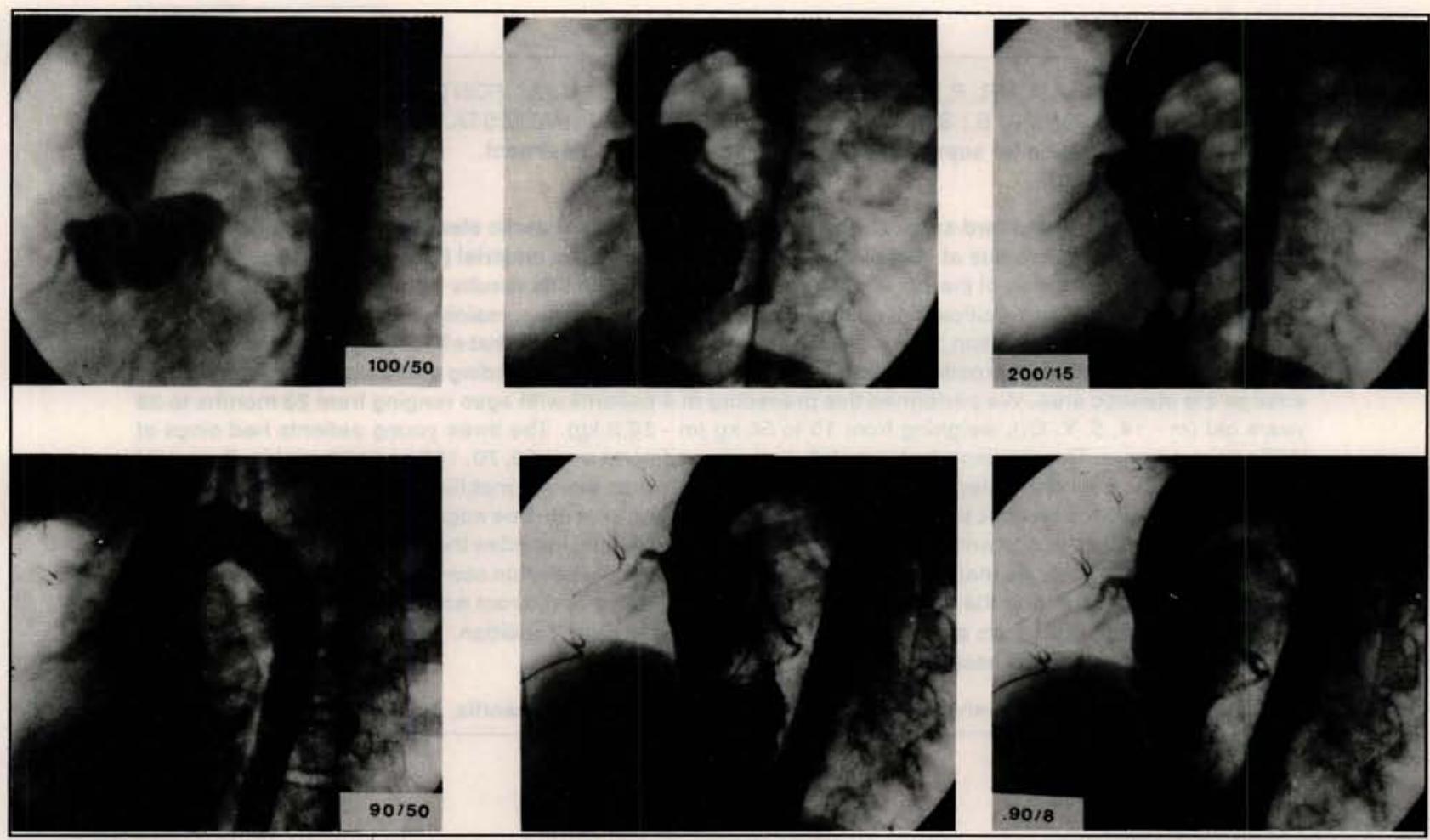

Fig. 7 - Aortograma e ventriculograma em diástole e sístole do caso de $n^{0} 4$. A série horizontal é pré-operatória e a inferior é pós-operatória.

estenosada utilizando material protético, e a ressecçāo por secção transversal da aorta com ampliaçāo dos três seios de Valsalva, também com material protético, seguida de reconstruçāo da aorta por plano de sutura horizontal.

Ainda que os poucos relatos sobre resultados com esses procedimentos sejam satisfatórios ${ }^{5,11,13}$, é possível prognosticarem-se alguns problemas na evoluçāo tardia, principalmente quando a cirúrgia é realizada em criança onde o enrijecimento dos tecidos protéticos empregados pode prejudicar o crescimento adequado da raíz da aorta.

$\mathrm{Na}$ técnica preconizada por $\mathrm{BROM}^{3}$, o volume de material protético nảo é pequeno, associado à sutura da aorta em um único plano, o que poderá provocar graus variáveis de estenose com o crescimento.

A proposição destas modificação técnica, por nāo necessitar de enxertos artificiais e realizar a sutura da aorta seguindo uma linha sinusoidal, evitaria qualquer possibilidade de reestenose na dependência dos dois fatores anteriormente citados.

Os bons resultados obtidos, ainda que a curto período de evolução pós-operatória, e apoiados nos aspectos anatômicos dos reestudos angiográficos (Figuras 6 e 7) permitem concluir ser esta técnica apropriada para a cirúrgia da estenose aórtica supravalvar, na sua forma localizada. 
SOUZA, L. C. B.; CHACCUR, P.; DINKHUYSEN, J. J.; FONTES, M. A.; FONTES, V. F.; ABDULMASSIH NETO, C.; ARNONI, A. S.; SILVA, M. V. D.; BOSíSIO, I. B. J.; PAULISTA, P. P.; JATENE, A. D. - Modificação técnica na cirurgia da estenose aórtica supravalvar. Rev. Bras. Cir. Cardiovasc., 7(2):121-126, 1992.

RBCCV 44205-170

SOUZA, L. C. B.; CHACCUR, P.; DINKHUYSEN, J. J; FONTES, M. A.; FONTES, V. F.; ABDUL MASSIH NETO, C.; ARNONI, A. S.; SILVA, M. V. D.; BOSÍSIO, I. B. J.; PAULISTA, P. P.; JATENE, A. D. - New tecnical modification for supravalvar aortic stenosis surgical treatment. Rev. Bras. Cir. Cardiovasc., 7 (2):121-126, 1992.

ABSTRACT: The standard surgical treatment of the supravalvar aortic stenosis is characterized by the ampliation of one or more sinus of Valsalva utilizing patch of prosthetic material (Dacron, bovine pericardium) with or without transsection of the aorta. One possible limitation on late results with these procedures is the fact of graft thickening or calcification, difficulting the aortic root growing, mainly when the patient is a child. In order to avoid this complication, we propose a new technical modification that effectivelly increases the aortic diameter, without the use of prosthetic material, using only the normal ascending aortic wall to reconstruct and enlarge the stenotic area. We performed this procedure in 4 patients with ages ranging from 23 months to 38 years old $(\mathrm{m}-14,5, Y, 0$,$) , weighing from 10$ to $56 \mathrm{~kg}(\mathrm{~m}-26,2 \mathrm{~kg})$. The three young patients had sings of Williams syndrome. The gradients between left ventricle and aorta were $50,70,100$ and $100 \mathrm{mmHg}$. Cristaloid cardioplegia was infunded antegrately. The ascending aorta was entirely mobilized. After aortic transsection immediately above the stenotic point, we made vertical incisions from the free edge to the botton of the Valsalva sinuses. Sometimes the size and position of the left coronary ostium impedes the incision on the left Valsalva sinus. On the distal aorta we made three longitudinal incisions in the position corresponding to the comissures of the aortic valve. Suturing these two parts we reconstructed the aortic root anatomicaly. The four patients had uneventful hospitalization and were discharged in good clinical condition. With a follow-up from 1 to 6 months, all the patients are assymptomatic.

DESCRIPTORS: supravalvar aortic stenosis, surgery; heart valve, aortic, surgery.

\section{REFERÊNCIAS BIBLIOGRÁFICAS}

1 BEUREN, A. J.; APITZ, J.; KONCZ, J. - Die Diagnose und Beurteilung derverschiedenen Formen der supravalvularen Aortenstenose. Ztschr. Kreislaufforsch., 51: 829-535, 1962.

2 BEUREN, A. J.; SCHULZE, C.; EBERLE, P.; HARMJANZ, D.; APITZ J. - The syndrome of supravalvular aortic stenosis, peripheral pulmonary stenosis, mental retardation and similar facial appearance. Am. J. Cardiol. 11: 471-483, 1964.

BROM, A. G. - Comunicado. In: KHONSARI (ed.). Atlas de cirurgia cardíaca. São Paulo, Livraria Editora Santos, 1990. p. 278-280.

DOTY, D. B.; POLANSKI, D. B.; JENSON, C. B. Supravalvular aortic stenosis: repair by extended aortoplasty. J. Thorac. Cardiovasc. Surg., 74: 362 371, 1977.

FLAKER, G.; TESKE, D.; KILMAN, J.; HOSIER, D.; WOOLEY, C. - Supravalvular aortic stenosis: a 20 year clinical perspective and experience with patch aortoplasty. Am. J. Cardiol., 51: 256-260, 1983.

HANCOCK, E. W. - Differentiation of valvar, subvalvar, and supravalvular aortic stenosis. Guy's Hosp. Rep., 110: 1, 1961.

HARA, M.; DUNGAN, T.; LINCOLN, B. - Supravalvular aortic stenosis: report of successful excision and aortic re-anastomosis. J. Thorac. Cardiovasc. Surg., 43: 212-221, 1962.
8 McGOON, D. C.; MANKIN, H. T.; VLAD, P.; KIRKLIN, J. W. - The surgical treatment of supravalvular aortic stenosis. J. Thorac. Cardiovasc. Surg. 41: 125133, 1961.

9 MURAD, H.; BASTOS, E.; BRITO, J. D.; JAZBIK, A. P.; NASCIMENTO, F. J.; GOMES, E. C.; FEITOSA, J. L. A.; BRAILE, D. M.; ARDITO, R. V. - Tratamento cirúrgico da estenose supra-aórtica por alargamento das áreas correspondentes aos seios de Valsalva com retalhos de pericárdio bovino. Arq. Bras. Cardiol., 55 (Supl. B) Resumo B 15, 1990.
10
PETERSON, T. A.; TODD, D. B.; EDWARDS, J. E. Supravalvular aortic stenosis. J. Thorac. Cardiovasc. Surg., 50: 734-741, 1965.

1 RASTELLI, G. C.; McGOON, D. C.; ONGLEY, P. A.; MANKIN, H. T.; KILKLIN, J. W. - Surgical treatment of supravalvular aortic stenosis. J. Thorac. Cardiovasc. Surg., 51: 873-882, 1966.

2 SHARMA, B. H.; FUJIWARA, H.; HALLMAN, G. L.; OTT, D. A.; REUL, G. J.; COOLEY, D. A. - Supravalvular aortic stenosis: 29 year review of surgical experience. Ann. Thorac. Surg., 51: 1031-1039, 1991.

3 STEWART, S.; ALEXSON, C.; MANNING, J. - Extended aortoplasty to relieve supravalvular aortic stenosis. Ann. Thorac. Surg., 46: 427-429, 1988.

4 WILLIAMS, J. C. P.; BARRATT-BOYES, B. G.; LOWE, J. B. - Supravalvular aortic stenosis. Circulation, 24: $1311-1318,1961$. 